\title{
Pregnancy from frozen-thawed blastocyst transfer increases risk of emergency cesarean section: a single-center retrospective study
}

\author{
Atsushi Yanaihara*, Shota Hatakeyama, Aguri Hirano, Makoto Ishikawa, Ryoma Taniguchi and Shirei Ohgi \\ Yanaihara Women’s Clinic, Kanagawa, Japan
}

\begin{abstract}
Backgroud: We aimed to investigate the incidence of emergency caesarean section (ECS) following problem-free frozen-thawed blastocyst transfer pregnancy.

Methods: Under 39years old of 1364 nulliparous patients (including 359 cases of ART pregnancy by frozen-thawed blastocyst transfer) after 37 gestational weeks, that were planned for singleton vaginal delivery and had problem-free progress of pregnancy, were retrospectively studied; they were divided into a normal vaginal delivery group and an ECS group. We used multivariate logistic regression modeling to examine the association between the mode of conception and several factors. 122 cases of ECS were divided into two subgroups: spontaneous conception (54cases) and ART pregnancy (68cases). Surgical indications were statistically classified between groups using Fisher's exact test. Surgical findings during ECS were compared.

Results: Statistical significance was observed in ART pregnancy (AOR 2.98, 95\% CI 1.78-5.00, P<0.001) and infant head circumference (AOR 1.72, 95\% CI 1.4$2.11, P<0.001)$. ECS was performed in $5.3 \%$ and $18.9 \%$ of the patients in the spontaneous conception and ART pregnancy groups, respectively. Protraction and arrest disorder were the commonest indication for ECS in both groups and was significantly higher after ART pregnancy than in spontaneous conception. There was no statistical difference between groups in the surgical findings.

Conclusions: The incidence of ECS in frozen-thawed blastocyst transfer pregnancy was three times higher than that in spontaneous conception. Protraction and arrest disorder were significantly higher in ART pregnancy, although it was the most frequent indication for ECS for both groups. This information is important for patient counseling.
\end{abstract}

\begin{abstract}
Abbreviations: ART: Assisted Reproductive Technology; CS: Cesarean Section; ECS: Emergency Cesarean Section; IVF: In Vitro Fertilization; ICSI: Intracytoplasmic Sperm Injection; HDP: Hypertensive Disorders of Pregnancy; NRFS: Non-Reassuring Fetal Status; AOR: Adjusted Odds Ratio.
\end{abstract}

\section{Introduction}

At present, the rate of cesarean sections (CS) differs in every country. Although Obstetrics and Gynecology societies of different countries differ in their guidelines as to the indications for CS, the ability to avoid CS depends on the on-site medical expertise during the delivery. Thus, patients who are capable of vaginal delivery may still deliver by CS depending on the parturient technique of the doctor who is present at delivery.

To date, there have been various reports on the perinatal outcome of in vitro fertilization (IVF)/intracytoplasmic sperm injection (ICSI) pregnancies. Many studies on ART pregnancy outcomes have reported high rates of CS [1-4] due to the increase in abnormalities of the placenta, such as placenta previa $[5,6]$. Many of these reports include cases of complications and premature delivery. The decision of emergency CS (ECS) is made during the natural progress of delivery. Although the indication of ECS include non-reassuring fetal status (NRFS), infections, and protraction and arrest disorder, there are no reports on the indications for ECS following ART pregnancy in the absence of prenatal complications. Furthermore, there are no comparison reports of surgical findings during ECS between ART pregnancy and spontaneous conception. The comparison of perioperative bleeding and quantity of oxytonic drugs may help to know the reaction on the uterus.

In this single-center retrospective study, we investigated the trends and characteristics of ECS following ART pregnancy, particularly for problem-free frozen-thawed blastocyst transfer.

\section{Patients and methods}

We retrospectively enrolled under 39years old 1364 cases of nulliparous (including359 cases of ART pregnancy) after 37 weeks of gestation that were planned for singleton vaginal delivery and had problem-free progress of pregnancy between September 2014 and March 2018. ECS was performed in 122 cases (54 spontaneous conception, 68 ART pregnancies). Breech deliveries, after myomectomy and prior CS were excluded in this study.

*Correspondence to: Atsushi Yanaihara MD, PhD, Yanaihara Women's Clinic, 1-26-29 Ofuna Kamakura Kanagawa Japan, 247-0056, E-mail: at123y@yahoo.co.jp

Key words: emergency cesarean section, ART, frozen-thawed blastocyst transfer, protraction and arrest disorder

Received: July 01, 2019; Accepted: July 09, 2019; Published: July 12, 2019 
This study was conducted with the approval of the Ethics Committee of Yanaihara Women's Clinic (YW/17-2), and written informed consent was obtained from all patients for this study.

All cases of ART pregnancies were induced by thawed embryo transfer of the blastocyst with hormone replacement cycle. The surgical team for ECS was limited to a few experts, and the procedures were performed by three experienced doctors. The indication for ECS was decided in accordance with the guidelines from the Japanese Society of Obstetrics and Gynecology. These included protraction and arrest disorder, NRFS, severe hypertensive disorders of pregnancy (HDP) and others such as infection. All cases were compared in terms of several factors, including maternal age, gestational age (weeks), maternal height, maternal weight at the last examination, infant weight, infant head circumference, and conception methods (spontaneous conception or ART pregnancy).

We used multivariate logistic regression modeling to examine the association between the mode of conception and the factors mentioned above. First, multivariate logistic regression models were estimated with the use of the mode of conception as the dependent variable for all putative explanatory variables. If the correlation between some variables chosen in the previous step was $\geq 0.8$ [7], these variables were excluded from further analysis. The model was presented with the adjusted odds ratio (AOR), 95\% confidence interval (CI), and $P$ value for each predictor.

The indications for ECS were compared for subgroup analysis between spontaneous conception and ART pregnancy using Fisher's exact test. To study the differences in surgical findings during ECS, all factors, including total bleeding, type of uterine contraction agent and concentration, and operative time, were compared between spontaneous conception and ART pregnancy with the Welch's test.

The amount of bleeding was calculated as the total bleeding from the operation start time to operation end time. We conducted all analyses using $\mathrm{R}$ version 3.3 .2 .1 . The standard $P<0.05$ was considered to be statistically significant in all tests.

\section{Results}

The normal vaginal delivery and ECS groups were compared with respect to the several factors mentioned above and the results are presented in Table 1. The multivariate logistic regression showed significant differences in infant head circumference (AOR 1.72, 95\% CI 1.4-2.11, $P<0.001$ ), and ART pregnancy (AOR 2.98, 95\% CI 1.78-5.00, $P<0.001$ ) (Table 2). Thus, bigger infant head circumference, and ART pregnancy were independent factors that increased the possibility of ECS.

Figure 1 shows the indications for ECS in spontaneous conception $(n=54)$ and ART pregnancy $(n=68)$. Protraction and arrest disorder were the commonest indication for ECS for both conception methods. NRFS and HDP were the next commonest indications for ECS in both groups. There was a significantly higher number of ECS for protraction and arrest disorder in the ART group than in the spontaneous conception group $(P<0.05)$.

The differences in operative findings during ECS for spontaneous conception and ART pregnancy are shown in Table 3. There were no differences in bleeding during operation, type of uterine contraction agent and concentration, and operative time between spontaneous conception and ART pregnancy.
Table 1. Comparison of factors between normal delivery and ECS

\begin{tabular}{|l|c|c|}
\hline & Normal delivery (n=1242) & ECS (n=122) \\
\hline Age (years) & $33.4 \pm 4.4$ & $35.1 \pm 4.0$ \\
\hline Gestational weeks (weeks) & $39.2 \pm 1.1$ & $39.6 \pm 1.2$ \\
\hline Height (cm) & $159.3 \pm 5.1$ & $158.0 \pm 5.8$ \\
\hline Weight at the last examination $(\mathrm{kg})$ & $61.2 \pm 7.2$ & $63.8 \pm 8.2$ \\
\hline Infant weight (g) & $3040.1 \pm 342.1$ & $3163.5 \pm 464.7$ \\
\hline Infant head circumference (cm) & $33.1 \pm 1.3$ & $34.0 \pm 1.7$ \\
\hline ART pregnancy & $23.5 \%$ & $55.7 \%$ \\
\hline
\end{tabular}

Data represented as mean $\pm \mathrm{SD}$ or number (percentage).

ECS, emergency cesarean section; ART, assisted reproductive technology

Table 2. Multivariate logistic regression modeling to examine the association between mode of pregnancy and several factors $(n=1364)$

\begin{tabular}{|l|c|c|}
\hline & Adjusted OR (95\% CI) & P value \\
\hline Infant head circumference $(\mathrm{cm})$ & $1.72(1.4,2.11)$ & $<0.001$ \\
\hline Method of conception & & \\
\hline Spontaneous pregnancy & $1[$ Reference $]$ & \\
\hline ART pregnancy & $2.98(1.78,5)$ & $<0.001$ \\
\hline
\end{tabular}

OR, odds ratio; CI, confidence intervals; ART, assisted reproductive technology

Table 3. Difference in surgical findings during ECS between spontaneous conception and ART pregnancy

\begin{tabular}{|l|c|c|c|}
\hline & Spontaneous (n=54) & ART $(\mathbf{n = 6 8})$ & P value \\
\hline Bleeding $(\mathrm{mL})$ & $631.6 \pm 427.2$ & $810.8 \pm 698.2$ & 0.135 \\
\hline Oxytocin $(\mathrm{U})$ & $3.9 \pm 3.2$ & $4.8 \pm 4.8$ & 0.298 \\
\hline Methylergometrine $(\mathrm{mL})$ & $0.36 \pm 0.38$ & $0.38 \pm 0.36$ & 0.766 \\
\hline Operation time (min) & $47.1 \pm 12.6$ & $46.7 \pm 14.3$ & 0.873 \\
\hline
\end{tabular}

Data represented as mean $\pm \mathrm{SD}$.

ECS, emergency cesarean section; ART, assisted reproductive technology

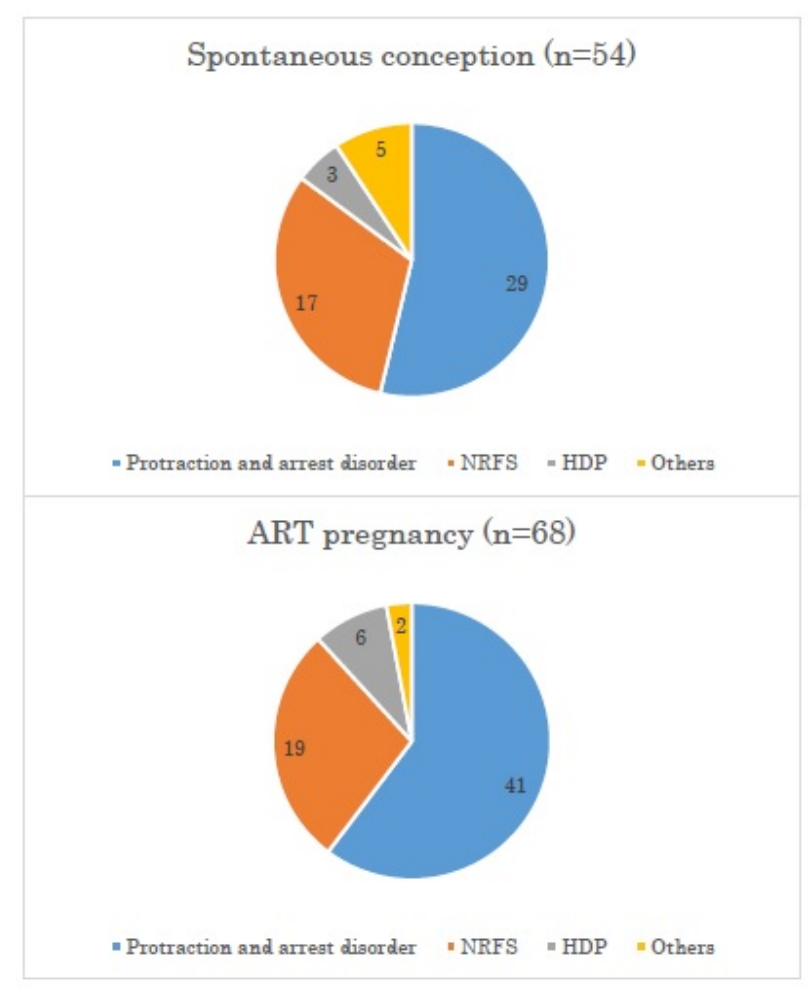

Figure 1. Indications for ECS in spontaneous pregnancy and ART pregnancy ECS, emergency cesarean section; ART, assisted reproductive technology; HDP, hypertensive disease of pregnancy; NRFS, non-reassuring fetal status 


\section{Discussion}

This study is the first report on ECS showing the comparison between ART pregnancy and spontaneous conception. According to this study, ART pregnancy is an independent risk factor for ECS and the incidence of ECS is 2.98 times higher in ART pregnancy than in spontaneous conception. The commonest indication for ECS was protraction and arrest disorder for both methods of conception; however, it was significantly higher in ART pregnancy than in spontaneous conception.

Meta-analyses on obstetric and perinatal outcomes from ART pregnancies have shown that ART pregnancy increases the risk of placenta previa, HDP $[4,8]$, gestational diabetes, and other complications $[1,5,9]$. The practice of CS has rapidly increased due to the increased number of complications in pregnancy. We excluded pregnancies in which a complication was diagnosed before term. Therefore, normal delivery was planned for all cases in this study before the onset of labor.

Although the probability of CS/ECS reportedly increases after ART pregnancy $[2,4,8-12]$, there are few reports on the practice of ECS for ART pregnancy without complications. According to the report from the Subcommittee of the Perinatology Committee of Japanese Society of Obstetrics and Gynecology [13], the rate of ECS for 239866 patients from 385 facilities was 15.3\%; however, the number of ECS after ART pregnancies is unclear. The report can be used for reference as it includes cases of preterm delivery and cases with complications.

In this study, it became clear that ART pregnancy is an independent risk factor for ECS. There are some reports of CS incidence after ART pregnancy [14]. When IVF and ICSI were compared, there was no significant difference in the CS rate [3]. One study showed no significant difference between frozen-thawed embryo transfer and fresh embryo transfer [4]. Another study, however, reported a higher risk of CS with frozen-thawed embryo transfer pregnancy than fresh embryo transfer pregnancy [15]. This was confirmed in another study, which showed that freezing resulted in higher risks of both elective CS and ECS [11]. However, these studies do not give a clear explanation as to the reason for the rise in the incidence of CS. The indications for elective CS and ECS are clearly different. Although our study included only normal fetal presentations, ART pregnancies may cause malposition of the placenta, which may influence the fetal position. Therefore, the probability of elective CS generally rises in the case of ART pregnancy.

It is well-known that parity and infant weight influence the delivery. In parous woman, labor is shorter than in primigravid women. If fetuses are bigger, the delivery tends to be more difficult. This study also demonstrated that infant head circumference significantly influenced the incidence of ECS.

Although there is no difference of newborn weight between spontaneous conception and an ART pregnancy according to this study and the report from the Subcommittee of the Perinatology Committee of Japanese Society of Obstetrics and Gynecology [13], the newborn weight is usually bigger in frozen-thawed embryo transfer pregnancy in Europe and America [16]. However, the mechanism is still unknown.

Protraction and arrest disorder is the commonest indication of ECS for both conception methods. Many situations can lead to the decision to perform ECS. Neumann et al. reported that vaginal deliveries are not increased when ART is used. Therefore, women undergoing ART may be in the early stage of labor when vaginal operative intervention is not possible [11]. A fixed notion that deliveries from ART pregnancies are difficult may influence the clinician's preference for CS over normal delivery. We have reported that medical interventions, such as use of labor-inducing drugs and instrumental delivery, are three times higher in ART pregnancy than in spontaneous conception [12]. Many of these indications are maternal rather than fetal in origin [12].

In this study, labor-inducing drugs such as oxytocin were used in many cases of ECS, and there was also reportedly an increase in inductions of labor in ART pregnancies [8,14]. Inadequate contractions can occur in both spontaneous and ART pregnancies. However, it seems that the incidence of protraction and arrest disorder is higher in IVF/ICSI than in spontaneous pregnancies. The response to uterotonic agents may be related to this. The myometrium is less responsive to uterotonic agents, such as oxytocin, with increasing maternal age [1719]. Women pregnant as a result of ART treatment may also exhibit a decreased response to uterotonic agents. Although the biological reason is still unknown, Arrowsmith et al. hypothesized that muscle atrophy and down-regulation of calcium channels may account for the decrease in uterine contractions with age [19]. Patients who are pregnant as a result of ART may have the same myometrial condition (or function) as that of ageing women. A study on the comparison of the biological mechanisms between the infertile and fertile myometrial functions is necessary to elucidate this hypothesis.

Inadequate contractility also affects perinatal bleeding [20]. There was no significant difference in bleeding during ECS between spontaneous and ARTI pregnancies in our study. In contrast, another study reported an increase in perinatal bleeding in ART pregnancies. Factors that increase uterine distention (infant weight, placental size, amniotic fluid volume) may predispose to bleeding due to uterine atony, in addition to poor uterine contractility. In Yanaihara's study, there was no significant differences in infant weight and placental weight between spontaneous and IVF/ICSI pregnancy [21]. In some aspects, CS procedures allow for greater control of the uterine contractions than during normal vaginal delivery; these include less retained placenta, control of uterine contractions with B-Lynch suture, and the direct administration of uterotonic agents such as methylergometrine. This may explain the different results in amounts of bleeding between vaginal delivery and CS delivery in ART pregnancies.

The reason for comparing the ECS findings, it is thought to be difference response of uterus contraction between spontaneous conception and ART pregnancy. There is no significant difference of bleeding between both groups means we can use enough uterotonic agents such as methylergometrine during the operation. In a word, it is supposed that the sensitivity for oxytocin may defective in ART pregnancy than spontaneous conception.

NRFS may be partly caused by velamentous insertion of the cord, the incidence of which was shown to be significantly increased in women with IVF pregnancies [21]. In particular, velamentous insertion of the cord located in the lower uterine segment should be deemed a high risk factor because variable decelerations without baroreceptormediated acceleration occur in such cases [22]. Although a velamentous insertion of the cord is not the only reason for NRFS, the risk of ECS may increase when it occurs.

We recognize that there are some limitations to our study. There is a possibility that parturient methods may be different depending on the progress of the delivery even with the same team and skill level. Additionally, the quantity of drugs, such as oxytocin, used for labor induction depends on the weight of the patient. However, due to medical insurance limits on the quantities of drug covered, the optimal amount of drug is not always used. If we can increase the oxytocin dose 
and increase the labor pain, ECS may decrease for both conception methods.

It is still unknown why ECS increases in cases of frozen-thawed blastocyst transfer pregnancy. Further studies to understand the relation between uterine muscle and ART is necessary.

In conclusion, our study showed that the incidence of ECS in ART pregnancy from frozen-thawed blastocyst transfer is three times higher than that in spontaneous pregnancy. The commonest indication for ECS was protraction and arrest disorder for both methods of conception; however, it was significantly higher in ART than in spontaneous conception. This information is important for patient counseling, and clinicians should be prepared for emergency. The reason for this was not clearly identified, but it could be related to the other individual physical characteristics of ART pregnancy such as less sensitive for oxytocin. Further studies are, thus, necessary to elucidate the reason for the higher incidence of ECS in ART pregnancies.

\section{Ethics approval and consent to participate}

This study was conducted with the approval of the Ethics Committee of Yanaihara Women's Clinic and with patient consent (YW/17-2)

\section{Consent for publication}

Written informed consent was obtained from the patient for publication of this study and any accompanying images. A copy of the written consent is available for review by the Editor of this journal.

\section{Competing interests}

The authors report no conflict of interest concerning the materials or methods used in this study or the findings specified in this paper. The authors have no competing financial interests related to this study.

\section{Authors' contributions}

AY drafted the manuscript.

$\mathrm{AH}, \mathrm{MI}$ and $\mathrm{AY}$ decided the parturient policy and participated in the deliveries.

$\mathrm{SH}$ and RT performed the statistical analyses.

SO helped to draft the manuscript.

\section{Declaration of funding and conflict of interest}

There is no funding source for this study.

The authors report no conflict of interest concerning the materials or methods used in this study or the findings specified in this paper. The authors have no competing financial interests related to this study.

\section{Acknowledgements}

The authors acknowledge the assistance of Editage for proofreading this manuscript.

\section{References}

1. Maman E, Lunenfeld E, Levy A, Vardi H, Potashnik G (1998) Obstetric outcome of singleton pregnancies conceived by in vitro fertilization and ovulation induction compared with those conceived spontaneously. Fertil Steril 70: 240-245. [Crossref]

2. Buckett WM, Chian RC, Holzer H, Dean N, Usher R, et al. (2007) Obstetric outcomes and congenital abnormalities after in vitro maturation, in vitro fertilization, and intracytoplasmic sperm injection. Obstet Gynecol 110: 885-891. [Crossref]
3. Yanaihara A, Yorimitsu T, Motoyama H, Ohara M, Kawamura T (2008) Clinical outcome of frozen blastocyst transfer; single vs. double transfer. $J$ Assist Reprod Genet 25: 531-534. [Crossref]

4. Maheshwari A, Pandey S, Shetty A, Hamilton M, Bhattacharya S (2012) Obstetric and perinatal outcomes in singleton pregnancies resulting from the transfer of frozen thawed versus fresh embryos generated through in vitro fertilization treatment: a systematic review and meta-analysis. Fertil Steril 98: 368-77.e1-9. [Crossref]

5. Esh-Broder E, Ariel I, Abas-Bashir N, Bdolah Y, Celnikier DH (2011) Placenta accreta is associated with IVF pregnancies: a retrospective chart review. BJOG 118: 1084 1089. [Crossref]

6. Iacovelli A, Liberati M, Khalil A, Timor-Trisch I, Leombroni M, et al. (2018) Risk factors for abnormally invasive placenta: a systematic review and meta-analysis. $J$ Matern Fetal Neonatal Med 22: 1-11. [Crossref]

7. Imdadullah M, Aslam M, Altaf S (2016) Mctest: an R package for detection of collinearity among regressors. $R J$ 8:495-505.

8. Toshimitsu M, Nagamatsu T, Nagasaka T, Iwasawa-Kawai Y, Komatsu A, et al (2014) Increased risk of pregnancy-induced hypertension and operative delivery after conception induced by in vitro fertilization/intracytoplasmic sperm injection in women aged 40 years and older. Fertil Steril 102: 1065-70.e1. [Crossref]

9. Sabban H, Zakhari A, Patenaude V, Tulandi T, Abenhaim HA (2017) Obstetrical and perinatal morbidity and mortality among in-vitro fertilization pregnancies: a population-based study. Arch Gynecol Obstet 296: 107-113. [Crossref]

10. Tomic V, Tomic J (2011) Neonatal outcome of IVF singletons versus naturally conceived in women aged 35 years and over. Arch Gynecol Obstet 284: 1411-1416. [Crossref]

11. Neumann K, Cirkel C, Rody A, Beyer DA (2017) Do ART patients face higher $\mathrm{C}$-section rates during their stage of delivery? A German monocenter experience. Arch Gynecol Obstet 295: 481-485. [Crossref]

12. Yanaihara A, Ohgi S, Motomura K, Hirano A, Takenaka S, et al. (2017) Medical intervention during labor increases after in vitro fertilization pregnancy. Clin Exp Obstet Gynecol XLIV(n): 6.

13. Kanayama N (2018) Subcommittee of the Perinatology Committee Report. p. 1504-37.

14. Pandey S, Shetty A, Hamilton M, Bhattacharya S, Maheshwari A (2012) Obstetric and perinatal outcomes in singleton pregnancies resulting from IVF/ICSI: a systematic review and meta-analysis. Hum Reprod Update 18: 485-503. [Crossref]

15. Zhao J, Xu B, Zhang Q, Li YP (2016) Which one has a better obstetric and perinatal outcome in singleton pregnancy, IVF/ICSI or FET?: a systematic review and metaanalysis. Reprod Biol Endocrinol 14: 51. [Crossref]

16. Roque M, Haahr T, Geber S, Esteves SC, Humaidan P (2019) Fresh versus elective frozen embryo transfer in IVF/ICSI cycles: a systematic review and meta-analysis of reproductive outcomes. Hum Reprod Update 25: 2-14. [Crossref]

17. Greenberg MB, Cheng YW, Sullivan M, Norton ME, Hopkins LM, et al. (2007) Does length of labor vary by maternal age? Am J Obstet Gynecol 197: 428.e1-7. [Crossref]

18. Main DM, Main EK, Moore DH, $2^{\text {nd }}$ (2000) The relationship between maternal age and uterine dysfunction: a continuous effect throughout reproductive life. Am J Obstet Gynecol 182: 1312-1320. [Crossref]

19. Arrowsmith S, Robinson H, Noble K, Wray S (2012) What do we know about what happens to myometrial function as women age? J Muscle Res Cell Motil 33: 209-217. [Crossref]

20. Yanaihara A, Motomura K, Hirano A, Takenaka S, Yanaihara T (2017) In vitro fertilization pregnancy is one of the risk factor for atonic bleeding in problem-free pregnancy. J Preg Child Health 4: 308.

21. Yanaihara A, Hatakeyama S, Ohgi S, Motomura K, Taniguchi R, et al. (2018) Difference in the size of the placenta and umbilical cord between women with natural pregnancy and those with IVF pregnancy. J Assist Reprod Genet 35: 431-434. [Crossref]

22. Hasegawa J, Matsuoka R, Ichizuka K, Sekizawa A, Okai T (2006) Velamentous cord insertion: significance of prenatal detection to predict perinatal complications. Taiwan J Obstet Gynecol 45: 21-25.

Copyright: (C2019 Yanaihara A. This is an open-access article distributed under the terms of the Creative Commons Attribution License, which permits unrestricted use, distribution, and reproduction in any medium, provided the original author and source are credited. 